\title{
Response of maize genotypes to gray leaf spot disease (Cercospora zeae-maydis) in the hills of Nepal
}

\author{
G Manandhar ${ }^{*}$, GO Ferrara**, TP Tiwari**, S Baidya*, \\ ASR Bajracharya ${ }^{*}$, BR Khadge ${ }^{*}$, and L Narro ${ }^{* *}$
}

\begin{abstract}
Gray leaf spot (GLS) is an important and destructive disease of maize in the hills of Nepal. The occurrence of this disease is recorded for the first time in the country in 2006. Several genotypes of maize supposed to be resistant to gray leaf spot in SARMP Zimbabwe and CIAT Colombia were evaluated together with other varieties in observation nursery conducted at farmer's fields as well as at Khumaltar (NARC research station in the Kathmandu Valley) during 2008 and 2009. Ten out of 28 genotypes of maize were identified as resistant to moderately resistant to GLS. The disease incidence was higher on the open pollinated varieties of maize. The severity of GLS on genotypes of maize observed as 3.0 and 2.2, respectively at Baluwapati and Dhungkharka in Kavrepalanchwok and 2.0 at Khumaltar in Lalitpur during 2008. The severity of GLS was observed as high as 2.4 at Pakhribas in Dhankuta and 1.7 at Khumaltar in 2009.
\end{abstract}

Key words: Maize, Nepal. Gray leaf spot, Cercospora zeae-maydis, resistant

\section{Introduction}

Gray leaf spot (GLS) disease caused by Cercospora zeae maydis Tehon \& Daniels is a serious threat to farmers and has caused serious losses in grain yield of maize in 2006. The disease was first identified from the state of Illinois, USA in 1925 (Tehon \& Daniels, 1925). Its occurrence was recorded in the Kavrepalanchowk district of Nepal (Manandhar, G. 2007). Grain yield losses of maize of up to 19 percent and 18 percent, respectively, on the local variety and on the improved OPV (Ganesh -1) were observed in field experiments (Manandhar and Baidya, 2010). The severity of GLS was high in the high hill region affecting adversely farmers who live with limited resources. The disease has been observed spreading over the years in 17 districts in the eastern, central and mid western regions of the country (Manandhar et al., 2009). GLS is evident on plants as small spots first on lower leaves of plants at tassel initiation. The disease moves upwards and spots change into long characteristics lesions within a month turning plants into a diseased field. The disease is significant since it rapidly destroys foliage when the plant is near at grain maturity. With objective to observe and measure reactions of maize to GLS, a nursery was grown in

\footnotetext{
* Plant Pathology Division, NARC. nepalgm@yahoo.com

** Hill Maize Research Program-International Maize and Wheat Improvement Center (CIMMYT/ Nepal)

*** CIMMYT, Colombia
} 
coordination with the District Agriculture Development Office (DADO). Seeds of maize cultivars with resistance to GLS in SARMP/CIAT/CIMMYT/Zimbabwe/Colombia were provided by HMRP/CIMMYT/N based in Kathmandu.

\section{Methodology}

Gray leaf spot nursery was planted at two different farmer's fields in Dhungkharka VDC-8 (2,040 masl) and Baluwapati VDC-7 (1,865 masl) in Kavrepalanchowk in 2008 and at Pakhribas VDC-7 (1,720 masl) in Dhankuta during 2009. The fields had the history of GLS epidemic in previous years. Sixteen to twenty eight genotypes of maize including OPVs, inbred line and other genotypes from CIMMYT's maize regional programs in Colombia and Zimbabwe were evaluated in two replications in RCBD with chemical fertilizer applied at 120:60:40 kg of NPK/ha. Other agronomical practices were followed as usual. The plot size for each genotype was 2-row of $3 \mathrm{~m}$ long at Baluwapati, and 2-row of $4 \mathrm{~m}$ long at Dhungkharka. Seeds of maize were sown at Baluwapati on $26^{\text {th }}$ of March and at Dhungkharka on $17^{\text {th }}$ of April in 2008. Twenty seven genotypes of maize was sown each in 2-row of $5 \mathrm{~m}$ long plot on 15 May in the observation nursery at Khumaltar (1,320 masl) in 2008. In the following year 13 genotypes of maize were sown each in 2-row of $4 \mathrm{~m}$ long on $2^{\text {nd }}$ of June at Khumaltar and 18 in 2-row of $3 \mathrm{~m}$ long on $8^{\text {th }}$ of April at Pakhribas. Due to long drought, seeds were re-sown for the second time on $9^{\text {th }}$ of June at Khumaltar. Ganesh-2 variety of maize being very susceptible to GLS was used as spreader and border rows to provide a congenial environment to GLS development at Khumaltar in 2009.

All genotypes of maize were scored for GLS at scale of 1-5, on one to three different dates at two weeks interval starting from the occurrence of lesions on leaves (i.e. on $10^{\text {th }}, 25^{\text {th }}$ of August and $1^{\text {st }}$ of September at Dhungkharka; $28^{\text {th }}$ of July, $12^{\text {th }}$ and $31^{\text {st }}$ of August at Baluwapati; $16^{\text {th }}$ of August and $5^{\text {th }}$ of September at Khumaltar in 2008; and during $30^{\text {th }}$ of August and $13^{\text {th }}$ of September at Khumaltar; and $13^{\text {th }}$ August at Pakhribas in 2009). The number of infected leaf was also recorded for the incidence of GLS. Agronomic characters such as days to tasseling, silking, plant height and ear height were recorded at Khumaltar. The weight of ears and moisture content of kernels were also recorded at Khumaltar on $9^{\text {th }}$ of September and on $1^{\text {st }}$ of October at Dhungkharka in 2008.

Gray leaf spot severity was rated according to Saghai Maroof et al., 1993, using following 1-5 scales:

1 = no symptoms;

$2=$ moderate lesion development below the leaf subtending the ear;

3 = heavy lesion development on and below the leaf subtending the ear with a few lesions above it;

$4=$ severe lesion development on all but the uppermost leaves, which may have a few lesions; and

5 = all leaves dead; 


\section{Results and discussion}

The disease began to appear on plants in mid July earlier on the local variety of maize at farmer's fields then in the nursery at higher elevation (1,720 to 2,040 masl) while at Khumaltar (1,320 masl) it appeared in mid to late August. The incidence of GLS was higher as 71 and 87 percent at severity of 2.7 and 2.8 on OPVs, respectively in the two years, while in Colombian material, it reached up to 67 percent at severity of 2.3 in 2008 and in the following year as 1.8 only on selected genotypes (Table 1). The reaction of disease on Zimbabwean maize was 2.2 and 1.8, respectively in two years. The incidence of GLS was highest as 75 percent at severity of 2.1 at Pakhribas in 2009. Ganesh-1, Deuti, Manakamana1, Manakamana-3 and Sitala were identified as moderately resistant OPVs, and Ganesh-2, local, S99TLWQ-HG-AB (Poshilo Makai-1), Manakamana-4, Manakamana-6 as susceptible OPVs. An inbred genotype of maize (source: CIMMYT/NMRP); TL 03A-S2 ElitexElite F2 1101-83 was found resistant to GLS with the lowest disease as 1.0 at Khumaltar and 1.4 at farmer's fields. GLS was lowest on Ganesh-1 and Manakamana -1 at Baluwapati; Manakamana-3, Manakamana-1, Sitala, Ganesh-1 and Deuti at Dhungkharka; Sitala, Ganesh-1 and Deuti at Pakhribas and Ganesh-1, Manakamana-1, Manakamana-3 and Deuti at Khumaltar. Three genotypes of maize including Manakamana -3 , Deuti and an inbred were observed as free of lesion in a preliminary field test in small plot of five plants of each for GLS at Khumaltar in 2007. This inbred also showed resistant reaction to GLS at farmer's field under epidemic condition at Kaleswor-9 VDC, Lalitpur in 2007 (Tiwari and Ferrara, 2007; PPD Annual report 2009). The inbred genotype of maize was possessing resistant reaction to northern leaf blight at NMRP and Khumal;tar since 2006. Similarly, Manakamana-1 and Ganesh-1 have been reported to have higher resistance over local at Panchthar and Solukhumbu, Terathum and Kavrepalanchowk (personal communication). Manakamana-3 and Deuti among distributed seven OPVs in relief program through DADO/NARC by MoA (Arun-2, Manakamana-1, Ganesh-2, Rampur Composite for mid hills and Ganesh-1, Manakamana-3 and Deuti for high hills) were observed moderately resistant to disease at Dhungkharka in 2007. Ganesh-1 (pool 9A), Manakamana-3 (Population 22) varieties were originally derived from population and pools of CIMMYT origin and that could be one of the major reason for being more resistant reaction over local in farmer's fields. Ganesh-1 was released for high hill region and other OPVs; Manakamana-3 (Pop. 22), Sitala (Pop. 44), Manakamana-4 (Pop. 45) and Deuti (ZM 621) for mid hill conditions.

CLA87/CLA91 and Corpoica H-112 genotypes introduced from Colombia were showing resistant reaction with the lowest severity level of GLS. Similarly, 05SADVI, ZM 525, ZM 627,07 SADVI genotypes were also resistant to GLS. A moderately resistant reaction with earliness in maturity was observed in ZM 501, ZM 305, ZM 309 and ZM 307. The mean days to produce tassel and silk were observed lowest as 74 and 77 on maize from Zimbabwe and highest as 77 and 81 on Colombian maize, while the mean of OPVs was intermediate as 75 and 77 at Khumaltar. Grain yield was highest in Manakamana-3 (4.050 t/h), 
CML451/CLO2450 (4.300 t/h) and ZM $305(4.250 \mathrm{t} / \mathrm{h})$ and ZM 501 at Khumaltar. Similarly, ZM 525 (5.564 t/h), ZM627, 07SADVI and ZM401 and Corpoica H-112 (4.058 $\mathrm{t} / \mathrm{h}$ ) among the new genotypes of maize produced the higher yield under disease epidemic conditions at Pakhribas (Table 2). Ganesh-1 (5.463 t/h) yielded highest at Pakhribas. The weight of ears from five randomly selected plants used for GLS score was highest in Manakamana-3, ZM 627 and Corpoica H-112 at Dhungkharka. Ear rot was remarkable at Baluwapati.

CML451/CLO2450 and ZM 305 among maize from SARMP, and Manakamana-6 and S99TLWQ-HG-AB (Poshilo Makai-1) among OPVs seemed to be tolerant to GLS. The short plant height, most preferred in hills was shortest on genotypes from Zimbabwe than that of the others. The local exhibited the tallest plant height and ear height as $271 \mathrm{~cm}$ and $157 \mathrm{~cm}$ among all maize. Host resistance for this worldwide important disease of maize has been reported on several hybrids and inbred lines (Hilty et al, 1979; Ward et al., 1999; Compendium of corn diseases 3rd edt., 1999). Details are given in Appendix 1 \& 2 .

\section{Conclusion}

Genotypes of maize resistant to moderately resistant to GLS was identified. The OPVs like Ganesh-1, Deuti, Manakamana-1 and Manakamana-3 (Ganesh-1 for high hills), can reduce yield loss in GLS prone environments of the hills. Several resistant genotypes and inbred maize can be useful for source of disease resistance in the national maize breeding program. Genotypes of maize including Corpoica H-112, CLA 87/CLA 91 from Colombia, and ZM 627, ZM 525, 05 SADVI, 07 SADVI, ZM 401 and ZM 501 from Zimbabwe were identified as resistant to GLS. Awareness to GLS, monitoring, crop improvement for GLS resistance and use of GLS resistant sources in the national maize breeding program should be emphasized.

\section{Acknowledgements}

This research is supported by NARC and HMRP/CIMMYT/N for which we are very thankful for the technical support, cooperation and seeds of maize. We are also thankful to the Chief of the Plant Pathology Division for necessary facilities and to the Chief of the Agriculture Research Station, Pakhribas for kind co-operation. We express our sincere thanks to Mr. D L Mandal, T-6, ARSP, and to all colleagues from PPD, ABD, NMRP, RARSL, CPDD, PRD, ORD for their sincere help. We are also grateful to Mr. IP Risal, ADO and Mr. M. K.C., Technician, ASC-Khopasi of Kvrepalanchowk and also to all the farmers including GP Sapkota, NR Dhakal, I Paudel and BN Timilsina in Dhungkharka; Baluwapati; Pakhribas and Kaleswor VDCs for their kind co-operation. We highly appreciate Ms. Laxmi Shrestha, Mr. S Shrestha including to all those who involved in experiments for their sincere help. 


\section{References}

Compendium of corn diseases (3rd edition). 1999. Edited by DG White, American Phytopathological Society, St. Paul Minnesota, USA Pp 22-23.

Hilty, JW, CH Hadden and FT Garden. 1979. Response of hybrids and inbred lines to gray leaf spot disease and the effects on yield in Tennessee. Plant Disease Reporter 63:515-518.

Manandhar, G and S Baidya. 2010. Gray leaf spot of maize, a new disease to Nepal and loss assessment. Proceedings of the 26th national summer crops workshop, national maize research program, Rampur, Chitwan, Nepal. 3-5 March 2010.

Manandhar, G. 2007. Gray leaf spot a threat to maize production in the hills of Nepal - in a quarterly newsletter of Nepal agricultural research council 14 (4) Oct.-Dec., Pp. 4

Manandhar, G, S Baidya, AR Bajracharya, GO Ferrara and TP Tiwari. 2009. Maize gray leaf spot disease - a report presented in review and planning meeting at NARI, Khumaltar, Nepal, organized by HMRP-III (NARC/DoA/SDC/CIMMYT). 14-16 Dec. 2009

PPD. 2009. Annual report plant pathology division, Nepal agricultural research council, Lalitpur, Nepal. F.Y. 2064/65 (2007-2008) Pp 50-52.

Maroof, S, MA Van, SW Scoyoc and YG Yu. 1993. Gray leaf spot disease of maize: Rating methodology and Inbre line evaluation. Plant Disease 77:583-587.

Tehon, LR and EY Daniels. 1925. Notes on the parasitic fungi of Illinois. Mycologia 17:240-249.

Tiwari, TP and GO Ferrara. 2007. Maize GLS monitoring - a trip report of hill maize research program- CIMMYT/N 13 September and 2-3 October 2007.

Ward, JMJ, EL Stromberg, DC Nowell and JrFW Nutter. 1999. Gray leaf spot: a disease of global importance in maize production'. Plant Disease 83:884-894. (from the Proceedings of the 9th Outreach Research Workshop at National Agriculture Research Institute, Khumaltar, Lalitpur, Nepal, June 7 - 8, 2010).

\section{Appendix 1. Gray leaf spot disease reaction on genotypes of maize from SARMP/ CIMMYT/Colombia/ Zimbabwe/NMRP at Dhungkharka, Baluwapati, Pakhribas and Khumaltar in 2008 and 2009}

1. a) OPVs

\begin{tabular}{|c|c|c|c|c|c|c|c|c|c|c|c|}
\hline \multirow{3}{*}{$\begin{array}{c}\mathbf{S} \\
\mathbf{N}\end{array}$} & \multirow{3}{*}{$\begin{array}{c}\text { Maize } \\
\text { Genotype }\end{array}$} & \multicolumn{4}{|c|}{ Incidence, \% } & \multicolumn{6}{|c|}{ Severity, 1-5 } \\
\hline & & \multirow{2}{*}{\begin{tabular}{c|}
$\mathrm{D} / \mathrm{rka}$ \\
'08
\end{tabular}} & \multirow{2}{*}{$\frac{\mathrm{B} / \mathrm{pti}}{\mathbf{0}}$} & \multirow{2}{*}{$\begin{array}{c}\text { Mn. } \\
\text { '08 }\end{array}$} & \multirow{2}{*}{$\frac{\mathrm{P} / \mathrm{bas}}{\mathbf{r} 09}$} & \multirow{2}{*}{$\frac{\mathrm{D} / \mathrm{rka}}{\mathbf{0} \mathbf{0 8}}$} & \multirow{2}{*}{$\frac{\mathrm{B} / \mathrm{pti}}{\mathbf{6} \mathbf{0 8}}$} & \multirow{2}{*}{$\begin{array}{c}\mathrm{K} / \mathrm{mal} \\
\mathbf{0 8}\end{array}$} & \multirow{2}{*}{$\begin{array}{c}\text { Mn. } \\
08\end{array}$} & \multicolumn{2}{|c|}{$\mathrm{K} / \mathrm{mal} \mathrm{P} / \mathrm{bas}$} \\
\hline & & & & & & & & & & '09 & '09 \\
\hline 1. & Mana-3 & 42 & 80 & 61 & 88 & 1.8 & 3.0 & 2.0 & 2.3 & - & 3.0 \\
\hline 2. & Sitala & 45 & 78 & 62 & 75 & 1.9 & 3.1 & 2.7 & 2.6 & - & 2.1 \\
\hline 3. & Mana-1 & 47 & 61 & 54 & 98 & 1.9 & 2.8 & 1.9 & 2.2 & - & 3.1 \\
\hline 4. & Deuti & 56 & 78 & 67 & 73 & 2.1 & 3.0 & 1.9 & 2.3 & - & 2.4 \\
\hline
\end{tabular}




\begin{tabular}{|c|c|c|c|c|c|c|c|c|c|c|c|}
\hline \multirow{3}{*}{$\begin{array}{l}\mathbf{S} \\
\mathbf{N}\end{array}$} & \multirow{3}{*}{$\begin{array}{c}\text { Maize } \\
\text { Genotype }\end{array}$} & \multicolumn{4}{|c|}{ Incidence, \% } & \multicolumn{6}{|c|}{ Severity, 1-5 } \\
\hline & & \multirow{2}{*}{$\begin{array}{c}\text { D/rka } \\
\text { '08 }\end{array}$} & \multirow{2}{*}{$\frac{\mathrm{B} / \mathrm{pti}}{\mathbf{6} \mathbf{0 8}}$} & \multirow{2}{*}{$\frac{\text { Mn. }}{{ }^{\prime} 08}$} & \multirow{2}{*}{$\begin{array}{c}\mathrm{P} / \mathrm{bas} \\
\mathbf{6} 09\end{array}$} & \multirow{2}{*}{$\frac{\mathrm{D} / \mathrm{rka}}{{ }^{\prime} \mathbf{0 8}}$} & \multirow{2}{*}{$\frac{\mathrm{B} / \mathrm{pti}}{{ }^{\prime} \mathbf{0 8}}$} & \multirow{2}{*}{$\begin{array}{c}\mathrm{K} / \mathrm{mal} \\
\mathbf{0 8}\end{array}$} & \multirow{2}{*}{$\begin{array}{c}\text { Mn. } \\
08\end{array}$} & \multicolumn{2}{|c|}{$\mathrm{K} / \mathrm{mal} \mathrm{P} / \mathrm{bas}$} \\
\hline & & & & & & & & & & ‘09 & ’09 \\
\hline 5. & Ganesh-1 & 54 & 81 & 68 & 77 & 2.1 & 2.8 & 1.7 & 2.2 & - & 2.4 \\
\hline 6. & Mana-6 & 79 & 87 & 83 & 100 & 3.0 & 3.5 & 2.7 & 3.1 & - & 2.8 \\
\hline 7. & Local & 74 & 84 & 79 & 100 & 2.9 & 3.5 & 3.3 & 3.2 & 2.2 & 2.8 \\
\hline 8. & Ganesh-2 & 85 & 97 & 91 & - & 3.2 & 3.8 & 3.0 & 3.3 & 2.7 & - \\
\hline 9. & Mana-4 & - & - & - & 79 & - & - & - & - & - & 2.9 \\
\hline 10. & S99TLWQ-HG-AB & - & - & - & 81 & - & - & - & - & - & 3.2 \\
\hline 11. & S99TLYQ-HG-AB & - & - & - & 96 & - & - & - & - & - & 2.8 \\
\hline & Mean & 60 & 81 & 71 & 87 & 2.4 & 3.2 & 2.4 & 2.7 & 2.5 & 2.8 \\
\hline
\end{tabular}

1. b) Maize genotype

\begin{tabular}{|c|c|c|c|c|c|c|c|c|c|c|c|}
\hline \multirow{3}{*}{$\begin{array}{c}\mathrm{S} \\
\mathbf{N}\end{array}$} & \multirow{3}{*}{ From Colombia } & \multicolumn{4}{|c|}{ Incidence, \% } & \multicolumn{6}{|c|}{ Severity, 1-5 } \\
\hline & & \multirow{2}{*}{$\frac{\mathrm{D} / \mathrm{rka}}{\mathbf{0} \mathbf{0 8}}$} & \multirow{2}{*}{$\frac{\mathrm{B} / \mathrm{pti}}{\mathbf{\prime} \mathbf{0 8}}$} & \multirow{2}{*}{$\begin{array}{c}\text { Mn. } \\
\text { '08 }\end{array}$} & \multirow{2}{*}{$\frac{\mathrm{P} / \mathrm{bas}}{\boldsymbol{\prime}^{\prime} \mathbf{0 9}}$} & \multirow{2}{*}{$\begin{array}{c}\text { D/rka } \\
\mathbf{0 8}\end{array}$} & \multirow{2}{*}{$\begin{array}{c}\text { B/pti } \\
\mathbf{0 8}\end{array}$} & \multirow{2}{*}{\begin{tabular}{|c|}
$\mathrm{K} / \mathrm{mal}$ \\
$\mathbf{0 8}$
\end{tabular}} & \multirow{2}{*}{$\begin{array}{c}\text { Mn. } \\
\mathbf{\prime} 08\end{array}$} & \multicolumn{2}{|c|}{$\mathrm{K} / \mathrm{mal} \mathrm{P} / \mathrm{bas}$} \\
\hline & & & & & & & & & & ‘09 & '09 \\
\hline 1. & Corpoica H-112 & 46 & 64 & 55 & 36 & 1.8 & 2.4 & 1.3 & 1.8 & 1.0 & 1.7 \\
\hline 2. & CLA 87/CLA 91 & 38 & 47 & 43 & 32 & 1.7 & 1.8 & 1.3 & 1.6 & 1.7 & 1.9 \\
\hline 3. & FNC 3056 & 51 & 83 & 77 & - & 2.1 & 3.3 & 1.3 & 2.2 & - & - \\
\hline 4. & ICA V-305 & 49 & 76 & 63 & - & 1.9 & 3.1 & 1.8 & 2.3 & - & - \\
\hline 5. & S03 TLYQ AB 05 & 66 & 69 & 68 & - & 2.6 & 2.9 & 1.5 & 2.3 & - & - \\
\hline 6. & CML 451/CLO2450 & 63 & 95 & 79 & - & 2.3 & 3.5 & 2.2 & 2.7 & 2.3 & - \\
\hline 7. & Cimcali $05 B$ roya 1 & 80 & 82 & 81 & - & 3.1 & 3.2 & 2.4 & 2.9 & - & - \\
\hline & Mean & 56 & 74 & 67 & 34 & 2.2 & 2.9 & 1.7 & 2.3 & 1.7 & 1.8 \\
\hline
\end{tabular}

1. c) Maize genotype

\begin{tabular}{|c|l|c|c|c|c|c|c|c|c|}
\hline \multirow{2}{*}{$\begin{array}{c}\text { S } \\
\mathbf{N}\end{array}$} & \multirow{2}{*}{$\begin{array}{c}\text { From } \\
\text { Zimbabwe }\end{array}$} & \multicolumn{2}{|c|}{ Incidence, \% } & \multicolumn{6}{|c|}{ Severity, 1-5 } \\
\cline { 3 - 10 } & & D/rka & P/bas & D/rka & K/mal & Mn. & K/mal & P/bas & Mn. \\
\cline { 3 - 10 } & & $\mathbf{0 8}$ & $\mathbf{0 9}$ & $\mathbf{0 8}$ & $\mathbf{0 8}$ & $\mathbf{0 8}$ & $\mathbf{0 9}$ & '09 & '09 \\
\hline 1. & 05SADVI & 26 & $\mathbf{5 7}$ & $\mathbf{1 . 5}$ & $\mathbf{1 . 0}$ & 1.2 & 1.2 & 2.0 & $\mathbf{1 . 6}$ \\
\hline 2. & ZM 525 & 35 & 71 & 1.7 & $\mathbf{1 . 0}$ & 1.4 & 1.4 & $\mathbf{1 . 9}$ & 1.7 \\
\hline 3. & ZM 627 & 26 & 61 & 1.6 & 1.7 & 1.7 & $\mathbf{1 . 1}$ & 2.0 & $\mathbf{1 . 6}$ \\
\hline 4. & 07SADVI & $\mathbf{2 4}$ & 80 & 1.6 & 1.7 & $\mathbf{1 . 7}$ & 1.7 & 2.0 & 1.9 \\
\hline 5. & ZM 401 & 27 & 78 & 1.7 & 2.1 & $\mathbf{1 . 9}$ & 1.2 & 2.2 & 1.7 \\
\hline 6. & 07SADVE & 40 & - & 2.0 & 1.9 & 2.0 & - & - & - \\
\hline
\end{tabular}




\begin{tabular}{|c|c|c|c|c|c|c|c|c|c|}
\hline \multirow{3}{*}{$\begin{array}{l}\mathbf{S} \\
\mathbf{N}\end{array}$} & \multirow{3}{*}{$\begin{array}{c}\text { From } \\
\text { Zimbabwe }\end{array}$} & \multicolumn{2}{|c|}{ Incidence, \% } & \multicolumn{6}{|c|}{ Severity, 1-5 } \\
\hline & & $\mathrm{D} / \mathrm{rka}$ & $\mathrm{P} / \mathrm{bas}$ & $\mathrm{D} / \mathrm{rka}$ & $\mathrm{K} / \mathrm{mal}$ & Mn. & $\mathrm{K} / \mathrm{mal}$ & $\mathrm{P} / \mathrm{b}$ as & Mn. \\
\hline & & '08 & ’09 & '08 & '08 & '08 & '09 & ’09 & '09 \\
\hline 7. & ZM 501 & 43 & 100 & 2.0 & 2.2 & 2.1 & 1.9 & 2.3 & 2.1 \\
\hline 8. & ZM 421 & 53 & - & 2.3 & 2.2 & 2.3 & - & - & - \\
\hline 9. & ZM 307 & 70 & - & 2.5 & 2.0 & 2.3 & - & - & - \\
\hline 10. & ZM 523 & 55 & - & 2.3 & 2.5 & 2.4 & - & - & - \\
\hline 11. & ZM 305 & 58 & - & 2.7 & 2.2 & 2.5 & 2.6 & - & - \\
\hline \multirow[t]{2}{*}{12.} & ZM 309 & 69 & - & 2.6 & 2.5 & 2.6 & - & - & - \\
\hline & Mean & 44 & 75 & 2.0 & 1.9 & 2.2 & 1.6 & 2.1 & 1.8 \\
\hline
\end{tabular}

\section{1. d) Maize inbred line from CIMMYT/NMRP}

\begin{tabular}{|c|c|c|c|c|c|c|c|c|}
\hline \multirow{3}{*}{$\begin{array}{l}\mathbf{S} \\
\mathbf{N}\end{array}$} & & \multicolumn{2}{|c|}{ Incidence, \% } & \multicolumn{5}{|c|}{ Severity, 1-5 } \\
\hline & & $\mathrm{D} / \mathrm{rka}$ & $\mathrm{B} / \mathrm{pti}$ & Mn. & $\mathrm{D} / \mathrm{rka}$ & $\mathrm{B} / \mathrm{pti}$ & Mn. & $\mathrm{K} / \mathrm{maL}$ \\
\hline & & ’08 & ’08 & ‘08 & ’08 & ’08 & ‘08 & ‘09 \\
\hline 1. & $\begin{array}{l}\text { TL 03A-S2 ElitexElite } \\
\text { F2 1101-83 }\end{array}$ & 14 & 30 & 22 & 1.3 & 1.5 & 1.4 & 1.0 \\
\hline
\end{tabular}

\begin{tabular}{|c|c|c|c|c|c|c|c|c|}
\hline \multirow{3}{*}{ GLE } & \multicolumn{3}{|c|}{ Incidence, \% } & \multicolumn{4}{c|}{ Severity, 1-5 } \\
\cline { 2 - 10 } & B/pti & D/rka & P/bas & B/pti & D/rka & K/maL & \multicolumn{2}{c|}{ P/bas K/mal } \\
\cline { 2 - 10 } & '08 & '08 & '09 & '08 & '08 & '08 & '09 & '09 \\
\hline Over all Mean & 75 & 51 & 77 & 3.0 & 2.2 & 2.0 & 2.4 & 1.7 \\
\hline
\end{tabular}

Appendix 2. Grain yields, days to 50 percent flowering, plant and ear height of genotypes of maize in gray leaf spot observation nursery at Khumaltar, Pakhribas and Dhungkharka in 2008 to 2009

2. a) Open Pollinated Varieties

\begin{tabular}{|c|l|c|c|c|c|c|c|c|c|c|}
\hline \multirow{2}{*}{ SN } & \multicolumn{1}{|c|}{$\begin{array}{c}\text { Maize } \\
\text { Genotypes }\end{array}$} & $\begin{array}{c}\text { Days to } \\
\text { Tassal }\end{array}$ & $\begin{array}{c}50 \% \\
\text { Silk }\end{array}$ & $\begin{array}{c}\text { Plant } \\
\text { ht.cm }\end{array}$ & $\begin{array}{c}\text { ear } \\
\text { ht.cm }\end{array}$ & $\begin{array}{c}\text { MC \% } \\
\text { K/mal }\end{array}$ & $\begin{array}{c}\text { GY.t/h } \\
\text { K/mal }\end{array}$ & $\begin{array}{c}\text { Fwt g } \\
\text { D/kra }\end{array}$ & $\begin{array}{c}\text { GY.t/h } \\
\text { K/mal }\end{array}$ & $\begin{array}{c}\text { GY.t/h } \\
\text { P/bas }\end{array}$ \\
\cline { 3 - 13 } & & 2008 & 2008 & 2008 & 2008 & 2008 & 2008 & 2008 & 2009 & 2009 \\
\hline 1. & Mana-3 & 78 & 81 & 241 & 137 & 32.5 & 4.050 & 900 & - & 4.226 \\
\hline 2. & Sitala white & 74 & 76 & 213 & 109 & 30.6 & 3.550 & 650 & - & 4.242 \\
\hline 3. & Mana-1 & 78 & 80 & 235 & 111 & 31.7 & 3.900 & 450 & - & 4.468 \\
\hline 4. & Deuti & 75 & 78 & 198 & 88 & 30.9 & 3.450 & 250 & - & 4.562 \\
\hline 5. & Ganesh-1 & 76 & 80 & 254 & 147 & 29.9 & 2.750 & 500 & - & 5.463 \\
\hline 6. & Ganesh-2 & 70 & 73 & 230 & 125 & 23.5 & 3.750 & 450 & 2.012 & - \\
\hline
\end{tabular}




\begin{tabular}{|r|l|c|c|c|c|c|c|c|c|c|}
\hline \multirow{2}{*}{ SN } & \multirow{2}{*}{$\begin{array}{c}\text { Maize } \\
\text { Genotypes }\end{array}$} & $\begin{array}{c}\text { Days to } \\
\text { Tassal }\end{array}$ & $\begin{array}{c}50 \% \\
\text { Silk }\end{array}$ & $\begin{array}{c}\text { Plant } \\
\text { ht.cm }\end{array}$ & $\begin{array}{c}\text { ear } \\
\text { ht.cm }\end{array}$ & $\begin{array}{c}\text { MC \% } \\
\text { K/mal }\end{array}$ & $\begin{array}{c}\text { GY.t/h } \\
\text { K/mal }\end{array}$ & $\begin{array}{c}\text { Fwt g } \\
\text { D/kra }\end{array}$ & $\begin{array}{c}\text { GY.t/h } \\
\text { K/mal }\end{array}$ & $\begin{array}{c}\text { GY.t/h } \\
\text { P/bas }\end{array}$ \\
\cline { 3 - 13 } & & 2008 & 2008 & 2008 & 2008 & 2008 & 2008 & 2008 & 2009 & 2009 \\
\hline 7. & Mana-6 & 72 & 74 & 222 & 112 & 25.9 & 3.500 & 730 & - & 3.986 \\
\hline 8. & Local & 74 & 77 & 271 & 157 & 28.8 & 3.900 & 750 & 1.990 & 3.390 \\
\hline 9. & S99TLWQ-HG-AB & - & - & - & & - & - & - & - & 4.468 \\
\hline 10. & S99TLYQ-AB & - & - & - & & - & - & - & - & 4.240 \\
\hline 11. & Mana-4 & - & - & - & - & - & - & - & - & 3.390 \\
\hline & & $\mathbf{7 5}$ & $\mathbf{7 7}$ & $\mathbf{2 3 3}$ & $\mathbf{1 2 3}$ & $\mathbf{2 9 . 2}$ & $\mathbf{3 . 6 0 6}$ & $\mathbf{5 8 5}$ & $\mathbf{2 . 0 0 1}$ & $\mathbf{4 . 2 4 4}$ \\
\hline
\end{tabular}

2. b) Genotypes of maize from Colombia

\begin{tabular}{|c|l|c|c|c|c|c|c|c|c|c|}
\hline 1. & CLA87/CLA91 & 76 & 78 & 222 & 122 & 31.9 & 3.800 & 550 & 2.663 & 3.003 \\
\hline 2. & FNC3056 & 80 & 82 & 198 & 99 & 32.8 & 3.250 & 450 & - & - \\
\hline 3. & CorpoicaH-112 & 78 & 80 & 216 & 111 & 30.2 & 3.900 & 700 & 1.874 & 4.058 \\
\hline 4. & ICAV-305 & 79 & 81 & 225 & 117 & 30.8 & 2.200 & 550 & - & - \\
\hline 5. & S03TLYQAB05 & 76 & 79 & 201 & 87 & 31.4 & 2.900 & 400 & - & - \\
\hline 6. & CML451/CLO2450 & 77 & 90 & 202 & 95 & 30.7 & 4.300 & 600 & 1.979 & - \\
\hline 7. & Cimcali05Broya1 & 74 & 77 & 180 & 87 & 30.0 & 3.050 & 600 & - & - \\
\hline & & $\mathbf{7 7}$ & $\mathbf{8 1}$ & $\mathbf{2 0 6}$ & $\mathbf{1 0 3}$ & $\mathbf{3 1 . 1}$ & $\mathbf{3 . 3 4 3}$ & $\mathbf{5 5 0}$ & $\mathbf{2 . 1 7 2}$ & $\mathbf{3 . 5 3 1}$ \\
\hline
\end{tabular}

\section{2. c) Genotypes of maize from Zimbabwe}

\begin{tabular}{|r|l|c|c|c|c|c|c|c|c|c|}
\hline 1. & 07SADVI & 75 & 78 & 203 & 109 & 29.1 & 3.950 & 550 & 2.905 & 4.913 \\
\hline 2. & ZM 501 & 70 & 73 & 201 & 92 & 28.0 & 3.550 & 530 & 3.194 & 4.240 \\
\hline 3. & 05SADVI & 76 & 78 & 218 & 111 & 31.6 & 4.150 & 750 & 2.622 & 3.780 \\
\hline 4. & ZM 627 & 77 & 80 & 212 & 107 & 31.6 & 3.600 & 900 & 2.762 & 5.536 \\
\hline 5. & ZM 525 & 76 & 79 & 179 & 79 & 30.8 & 3.100 & 800 & 2.214 & 5.564 \\
\hline 6. & ZM 305 & 70 & 73 & 195 & 89 & 28.8 & 4.250 & 430 & 1.691 & - \\
\hline 7. & 07SADVE & 75 & 77 & 177 & 75 & 30.7 & 3.250 & 750 & - & - \\
\hline 8. & ZM 309 & 69 & 72 & 174 & 78 & 26.9 & 2.800 & 300 & - & - \\
\hline 9. & ZM 523 & 76 & 79 & 216 & 114 & 30.8 & 3.000 & 680 & - & - \\
\hline 10. & ZM 421 & 78 & 80 & 221 & 111 & 29.3 & 3.200 & 500 & - & - \\
\hline 11. & ZM 401 & 73 & 76 & 205 & 97 & 28.8 & 4.000 & 500 & 1.993 & 4.258 \\
\hline 12. & ZM 307 & 70 & 73 & 190 & 82 & 27.1 & 3.050 & 700 & - & - \\
\hline & & $\mathbf{7 4}$ & $\mathbf{7 7}$ & $\mathbf{1 9 9}$ & $\mathbf{9 5}$ & $\mathbf{2 9 . 5}$ & $\mathbf{3 . 4 9 2}$ & $\mathbf{6 1 6}$ & $\mathbf{2 . 4 8 3}$ & $\mathbf{4 . 7 1 5}$ \\
\hline
\end{tabular}




\section{2. d) Maize inbred genotype of maize from CIMMYT/NMRP}

\begin{tabular}{|l|c|c|c|c|c|c|c|c|}
\hline $\begin{array}{l}\text { TL 03A-S2 } \\
\text { ElitexElite F2 }\end{array}$ & $\mathbf{1 1 0 1}$ & $\mathbf{8}$ & - & - & - & - & - & 2.199 \\
\hline Over all Mean & $\mathbf{7 5}$ & $\mathbf{7 8}$ & $\mathbf{2 1 1}$ & $\mathbf{1 0 5}$ & $\mathbf{2 9 . 8}$ & $\mathbf{3 . 4 8 7}$ & $\mathbf{5 6 9}$ & $\mathbf{2 . 3 1 5}$ \\
\hline
\end{tabular}

Abbreviations: '_' : not included; Mana : Manakamana; S99TLWQ-HG-AB: Poshili Makai-1 D/rka : Dhungkharka; B/pti : Baluwapati; P/bas: Pakhribas; K/mal: Khumaltar; ht.: height; cm: centimeter; Days to 50\% : days required to tassel/silk in $50 \%$ of plants; MC: moisture content of kernels; g: gram; $\mathrm{t} / \mathrm{h}$ : ton per hectare; GY: grain yield adjusted at 14 percent mc; Fwt : Fresh weight of ears from five plants; '08 : 2008; '09: 2009; Mn.: Mean; masl: meter above sea level; SARMP: South American Regional Maize Program; CIMMYT: International Maize and Wheat Improvement Center; NMRP: National Maize Research Program. 